
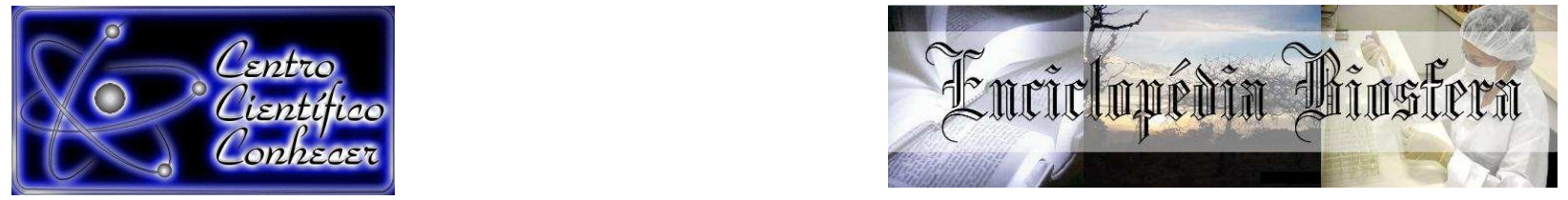

\title{
INCREMENTO POTENCIAL EM ÁREA TRANSVERSAL PARA ARAUCÁRIA
}

\author{
Emanuel Arnoni Costa ${ }^{1}$, César Augusto Guimarães Finger ${ }^{2}$ \\ ${ }^{1}$ Doutor em Manejo Florestal, Universidade Federal de Santa Maria \\ (emanuelarnonicost@hotmail.com) \\ ${ }^{2}$ Prof. Dr. do Departamento de Engenharia Florestal, Universidade Federal de Santa \\ Maria
}

Recebido em: 03/10/2016 - Aprovado em: 21/11/2016 - Publicado em: 05/12/2016 DOI: 10.18677/EnciBio_2016B_056

\begin{abstract}
RESUMO
O incremento periódico anual potencial em área transversal de árvores de Araucaria angustifolia (Bertol.) Kuntze de crescimento livre foi determinado usando a metodologia proposta por Bragg (2001). Um total de 183 araucárias foram medidas no ano de 2013 nos municípios de Canoinhas (SC) e Lages (SC). Cada árvore teve amostrados dois rolos de incremento extraídos radialmente do tronco ao nível do diâmetro à altura do peito utilizando o trado de Pressler. Um modelo não linear foi ajustado para descrever o incremento potencial. Os resultados indicam o limite potencial de incremento periódico em área transversal de araucária em área de ocorrência natural.
\end{abstract}

PALAVRAS-CHAVE: Análise de regressão, Crescimento máximo, Pinheiro-doparaná.

\section{POTENTIAL INCREMENT IN BASAL AREA OF ARAUCARIA}

\begin{abstract}
The potential annual periodic increment in basal area free growth trees of Araucaria angustifolia (Bertol.) Kuntze was determined using the methodology proposed by Bragg (2001). A total of 183 araucaria were measured in the year 2013 in the municipalities of Canoinhas (SC) and Lages (SC). Each tree had sampled two increment rolls radially extracted from the trunk at the level of diameter at breast height using the Pressler borer. A non-linear model was fitted to describe the potential increase. The results indicate the potential limit of periodic increment in basal area of Araucaria in natural occurrence.
\end{abstract}

KEYWORDS: Parana-pine; Regression analysis; Maximum growth.

\section{INTRODUÇÃO}

Na região Sul do Brasil, uma das formações florestais mais características do Bioma Mata Atlântica, corresponde a Floresta Ombrófila Mista (OLIVEIRA-FILHO \& FONTES, 2000), também conhecida como "Floresta de Araucária" (IBGE, 2012) dominada pela Araucaria angustifolia (Bertol.) Kuntze que constitui o dossel superior (HIGUCHI et al., 2012). Nesta Floresta também se destacam outras espécies, tais como: Ilex paraguariensis A. St.-Hil., Mimosa scabrella Benth., Podocarpus lambertii Klotzsch ex Endl., Ocotea spp., Nectandra spp., entre outras (SILVA et al., 2012; HIGUCHI et al., 2013; NEGRINI et al., 2014). 
As Florestas de Araucária cobrem grandes extensões de terras principalmente nos três estados do sul do Brasil: Rio Grande do Sul, Santa Catarina e Paraná (OLIVEIRA-FILHO et al., 2013), também está presente em algumas áreas em São Paulo, Rio de Janeiro, Minas Gerais, bem como em pequenas áreas da província de Missiones, na Argentina e Paraguai (IRIARTE \& BEHLING, 2007).

Para manejar florestas, modelos de crescimento e produção são ferramentas importantes para o planejamento, pois possibilitam simular o desenvolvimento do povoamento e produção sob várias alternativas de manejo (VANCLAY, 1994). Árvores livres que crescem sem a influência de árvores competidoras podem apresentar crescimento e dimensão potencial para algumas variáveis morfométricas, constituindo um referencial decisivo quanto ao limite da espécie, quando comparado a árvores crescendo sob condições de concorrência na floresta.

Nesse contexto, é importante conhecer o comportamento do crescimento e suas modificações interdimensionais em árvores de crescimento livre (Hasenauer, 1997), uma vez que auxiliam as estratégias para a elaboração de guias de manejo florestal. Na modelagem do crescimento florestal, árvores livres são usadas para descrever o crescimento potencial (WEISKITTEL et al., 2011).

A respeito deste conceito de modelagem, o crescimento potencial busca definir o limite atingido em níveis de taxas de crescimento para uma espécie com base em suas características dimensionais: diâmetro, altura, altura de inserção da copa, comprimento de copa, proporção de copa, diâmetro de copa, entre outros (Hasenauer, 2006). Ao contrário, a função modificadora consiste em um componente de declínio do crescimento potencial, normalmente decorrente da modelagem de árvores situadas na floresta, levando em consideração múltiplas influências ambientais: competição entre árvores, densidade do povoamento, limite de recursos, entre outros fatores (QUICKE et al., 1994).

Desta forma, o presente estudo teve como objetivo definir uma equação para determinar o incremento periódico anual potencial em área transversal de árvores de Araucaria angustifolia (Bertol.) Kuntze, crescendo livre, em campo aberto, em locais de ocorrência natural na região Sul do Brasil.

\section{Áreas de estudo}

\section{MATERIAL E MÉTODOS}

Árvores crescendo livre de competição foram amostradas no ano de 2013 nos municípios de Canoinhas (SC) e Lages (SC). Para os municípios estudados, o clima, de acordo com a classificação de Köppen, é subtropical úmido, sem estação seca e com verão temperado (Cfb). Na Tabela 1, são apresentadas as coordenadas geográficas dos municípios estudados, a altitude média em relação ao nível médio do mar, em $\mathrm{m}$, a temperatura anual média (TAM), em ${ }^{\circ} \mathrm{C}$, e a precipitação anual média (PAM), em mm (Alvares et al., 2013).

TABELA 1 - Localização e características climáticas das áreas estudadas

\begin{tabular}{cccccc}
\hline Municípios & Latitude & Longitude & Altitude $(\mathbf{m})$ & TAM & PAM \\
\hline Canoinhas & $-26^{\circ} 10^{\prime}$ & $-502^{\prime}$ & 831,0 & 17,2 & 1607,4 \\
Lages & $-27^{\circ} 48^{\prime}$ & $-50^{\circ} 9^{\prime}$ & 987,0 & 15,2 & 1684,7 \\
\hline
\end{tabular}

Em que: TAM = temperatura anual média, em ${ }^{\circ} \mathrm{C} ; \mathrm{PAM}=$ precipitação anual média, em $\mathrm{mm}$. 


\section{Levantamento dos dados}

As árvores de araucária foram amostradas de forma intencional, visando cobrir toda amplitude de distribuição diamétrica que ocorrem nos locais estudados. Assim, 67 araucárias foram amostradas em Canoinhas (SC) e 116 em Lages (SC). Em cada árvore, foram medidos: o diâmetro à altura do peito (DAP) com fita diamétrica, a altura total (h) com hipsômetro Vertex IV, e extraídos rolos de incrementos, usando o trado de Pressler na altura do diâmetro à altura do peito.

Dois rolos de incremento de cada árvore foram acondicionados em canudos de plástico perfurados e identificados com o número da árvore. Em laboratório as amostras de rolos de incrementos foram coladas em suportes de madeira. Após a secagem, as amostram foram preparadas com lixadeira manual com intuito de tornar mais fácil a visualização dos anéis de crescimento.

$O$ incremento foi delimitado anualmente, pelos anéis de crescimento, nos rolos em um período que compreendeu os últimos cinco anos. Após a delimitação dos anéis de crescimento, as amostras foram digitalizadas com scanner, e as imagens armazenadas em arquivo com formato imagem com resolução de 1200 pixels. Com a digitalização, os anéis de crescimento foram medidos em milímetros, com o Software Image Pro-Plus, registrando-se os incrementos radiais de cada uma das árvores em arquivo de texto.

\section{Incremento periódico potencial em área transversal}

O incremento periódico anual potencial em área transversal de árvores de crescimento livre foi determinado ajustando o modelo proposto por Bragg (2001):

$$
\operatorname{IPAg}(\%)=\beta_{1} \cdot \text { DAP }_{-t}^{\beta 2} \beta_{3}{ }^{\text {DAP-t }}+\varepsilon
$$

Em que: IPAg $(\%)=\left\{\left[\left(g_{-} g_{-t}\right) / g_{-t}\right]^{*} 100\right\} / t=$ incremento periódico anual potencial em área transversal; $g=\pi \cdot \mathrm{DAP}^{2} / 4 ; \mathrm{g}_{-\mathrm{t}}=\pi \cdot \mathrm{DAP}_{-\mathrm{t}} / 4 ; \mathrm{DAP}=$ diâmetro à altura do peito no final do período, em $\mathrm{cm}$; $\mathrm{DAP}_{-\mathrm{t}}=$ diâmetro à altura do peito no início do período, em $\mathrm{cm} ; \mathrm{t}=$ número de anos considerados (últimos cinco anos); $\varepsilon=$ erro residual.

Para descrever o IPAg (\%), foi obtido um subconjunto de dados distribuídos ao longo de toda a amplitude de classes diamétricas, que permitiu definir o limite quanto ao potencial de crescimento nos locais investigados.

\section{Análise dos dados}

Todas as estatísticas foram processadas no Sistema de Análise Estatística no programa $R$ (R DEVELOPMENT CORE TEAM, 2013). O teste t para duas amostras independentes foi usado para comparar as médias de altura entre os dois municípios estudados. Esta análise só foi possível, após verificar que as alturas seguiam distribuição normal, de acordo com o teste de Kolmogorov-Smirnov. Os critérios usados para verificar a performance do ajuste do modelo de regressão foram o coeficiente de determinação $\left(R^{2}\right)$ e o erro padrão da estimativa (Syx). Para o desenvolvimento de gráficos empregou-se a planilha eletrônica Excel (Microsoft ${ }^{\Theta}$ Office, 2013).

\section{RESULTADOS E DISCUSSÃO}

A amplitude de distribuição diamétrica de árvores de crescimento livres amostradas foi de 14,5 a $104,6 \mathrm{~cm}$, considerando todo o conjunto de dados (Tabela 2). Nas áreas de campo aberto, encontrou-se dificuldade para encontrar araucárias 
livres de competição com grande porte (DAP $>70,0 \mathrm{~cm}$ ), acreditando-se que a maioria dos exemplares tenha sido cortada devido à expansão de áreas para atividades de pecuária e agricultura.

TABELA 2 - Resumo estatístico das características dendrométricas de árvores de araucária crescendo livre.

\begin{tabular}{cccccccc}
\hline Variável & Município & $\mathbf{n}$ & Mínimo & Média $^{*}$ & Mediana & Máximo & CV\% \\
\hline DAP & & & 14,5 & 37,3 & 37,2 & 92,3 & 38,0 \\
DAP-t & Canoinhas (SC) & \multirow{2}{*}{67} & 7,6 & 30,9 & 31,5 & 91,5 & 51,6 \\
H & & & 8,3 & $15,7 \mathbf{a}$ & 16,3 & 23,4 & 23,9 \\
\hline DAP & \multirow{2}{*}{ Lages (SC) } & \multirow{2}{*}{116} & 18,0 & 42,1 & 41,1 & 104,6 & 28,1 \\
DAP-t & & 7,1 & 37,6 & 36,8 & 104,0 & 31,8 \\
H & & & 7,3 & $12,6 \mathbf{b}$ & 12,3 & 19,4 & 21,3 \\
\hline DAP & \multirow{2}{*}{ Todos } & \multirow{2}{*}{183} & 14,5 & 40,4 & 39,9 & 104,6 & 32,0 \\
DAP-t & & 7,6 & 35,2 & 34,3 & 104,0 & 39,5 \\
H & & & 7,3 & 13,7 & 13,3 & 23,4 & 25,1 \\
\hline
\end{tabular}

Em que: $\mathrm{n}=$ número de árvores medidas; $\mathrm{CV} \%$ = coeficiente de variação; $\mathrm{DAP}=$ diâmetro à altura do peito no final do período, $\mathrm{em} \mathrm{cm} ; \mathrm{DAP}_{-\mathrm{t}}=$ diâmetro à altura do peito no início do período, em $\mathrm{cm} ; \mathrm{h}=$ altura total, em $\mathrm{m}$. Teste de normalidade de Kolmogorov-Smirnov para a variável $\mathrm{h}$ nos dois municípios; Canoinhas - $(\mathrm{D}=$ 0,0926; $\operatorname{Pr}>\mathrm{D}=>0,150)$; Lages $-(\mathrm{D}=0,0698, \operatorname{Pr}>\mathrm{D}=>0,150) .{ }^{*}$ Teste t para duas amostras independentes, segundo a variável $h$. Médias com a mesma letra na coluna não diferem entre si $(\alpha=5 \%)$.

Ao avaliar as alturas de árvores livres, segundo o teste $t$, foi observada diferença significativa entre as médias de $\mathrm{h}$ de araucárias amostradas no município de Canoinhas (SC), quando comparado a Lages (SC) $(t=-6,52, p<0,0001)$. Árvores livres de Canoinhas (SC) apresentam $\mathrm{h}$ média de 15,7 m, com valor médio 3,1 $\mathrm{m}$ maior que em Lages (SC), onde alcançou a média de 12,6 m. Essa diferença pode estar associada a condições específicas de sítios dos locais, decorrente da combinação dos fatores ambientais, físicos e biológicos.

Alguns problemas com perturbações espaciais e temporais na produtividade do sítio florestal em ambiente natural podem ser de difícil solução, tanto no inventário, como nos procedimentos de modelagem, sendo recomendado uma modelagem adaptável, com parâmetros específicos do sítio, que podem ser atualizados a qualquer momento, quando novas informações se tornem disponíveis (SKOVSGAARD \& VANCLAY, 2013). Isso ajudará a garantir o uso eficiente de informações importantes, incluindo possíveis alterações na produtividade local e desempenho de crescimento.

A produtividade e as interações entre as espécies são influenciadas pela disponibilidade de diferentes recursos e condições climáticas, todos os quais mudam espacialmente e temporalmente. Os modelos são uma ferramenta valiosa para prever como esses fatores interagem e podem influenciar o crescimento e outras funções e serviços (PRETZSCH et al., 2015).

Recentemente, em abordagens sobre a previsão da produtividade de sítios florestais; BONTEMPS \& BOURIAUD (2014) sugeriram incluir informações espaciais sobre a estrutura genética das populações e as suas interações com o ambiente abiótico. Estes aspectos continuam sendo um grande desafio quanto a compreensão das variações espaciais na produtividade da área de floresta, em escala ampla e, no 
fornecimento de uma base genética à silvicultura, adaptadas às alterações climáticas.

As características de clima para os municípios estudados (Tabela 1) comprovam que a altitude e a temperatura anual média (TAM) são as variáveis que mais diferenciam entre os dois locais, mas com valores parecidos de precipitação média anual (PAM). Em regiões de maior altitude, a $\mathrm{h}$ das árvores tende a diminuir, o que ocorreu em Lages (SC) quando confrontado a Canoinhas (SC). Esta é uma relação conhecida em áreas de florestas temperadas, onde as árvores crescem menos em altitudes mais elevadas (COOMES \& ALLEN, 2007; KING et al., 2013) e apresentam alturas inferiores (PAULSEN et al., 2000). O ajuste do modelo (1) definiu uma linha de referência para o crescimento potencial com os dados do presente estudo (Figura 1).

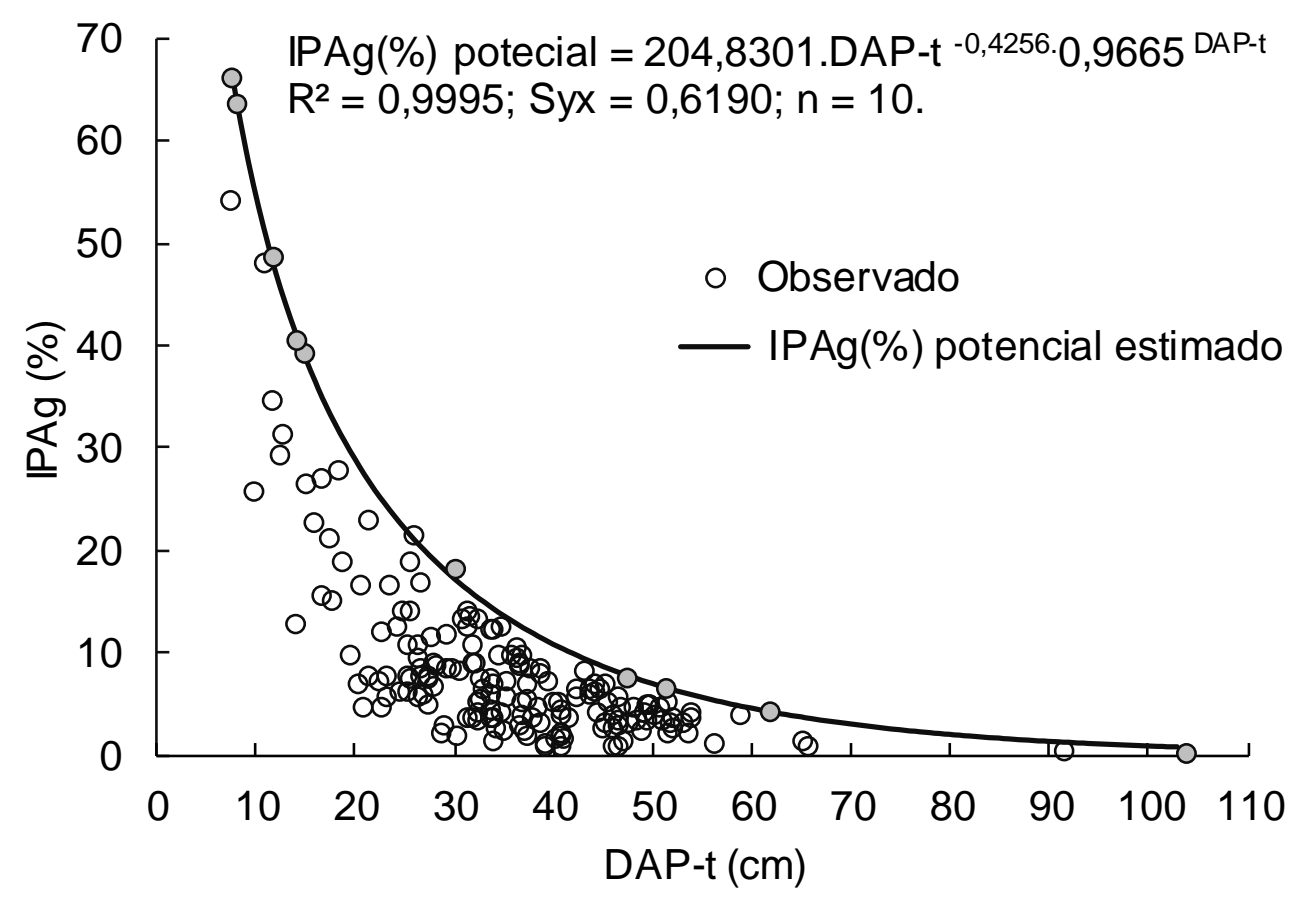

FIGURA 1 - Relação entre IPAg (\%) potencial estimado em função do diâmetro à altura do peito no início do período (DAP-t).

Esta linha independe de fatores inerentes ao sítio, já que os dados das árvores utilizados no ajuste pertenciam a um subconjunto de 10 árvores de araucária distribuídas ao longo de toda amplitude de DAP-t e representavam os níveis máximos das taxas de crescimento. BRAGG (2003) mostrou que, normalmente, o conjunto de dados usado para o ajuste desta relação contém entre 6 a 12 árvores. A equação ajustada apresentou valor de coeficiente de determinação $\left(R^{2}=0,9995\right)$ e erro padrão da estimativa $($ Syx $=0,6190)$.

Dessa forma, a taxa máxima de IPAg encontrada foi $139,2 \mathrm{~cm}^{2} /$ ano para um DAP-t $=46,0 \mathrm{~cm}$. As linhas com reduções: $75 \%, 50 \%, 25 \%$ e $5 \%$ da tendência do IPAg potencial foram arbitradas com intuito de obter números indicadores quanto ao crescimento de araucária, ao longo da amplitude de distribuição diamétrica amostrada (Figura 2). 


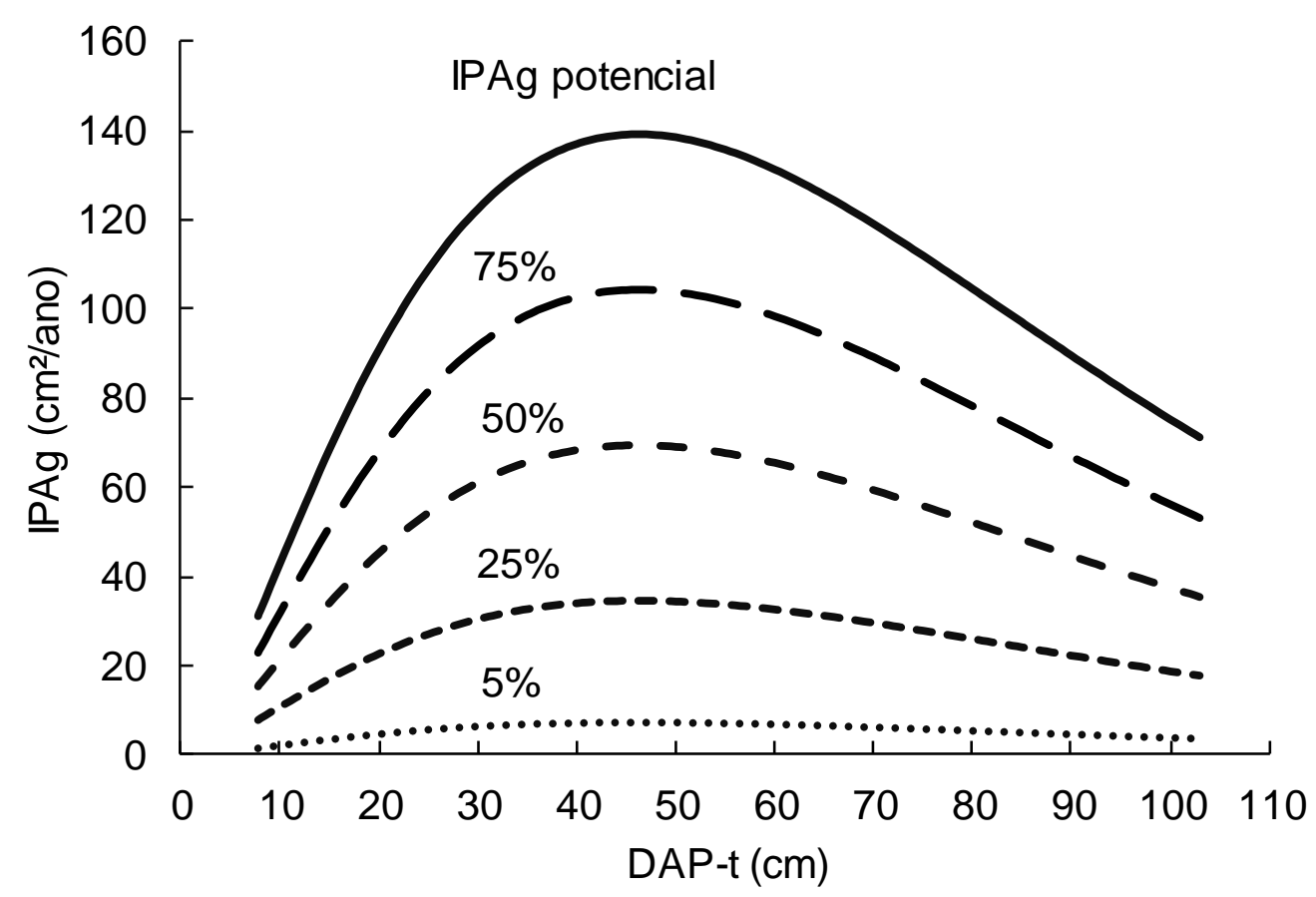

FIGURA 2 - Incremento periódico anual em área transversal (IPAg) potencial e reduções preestabelecidas em: $75 \%$; $50 \%$; $25 \%$; $5 \%$; em função do diâmetro à altura do peito no início do período (DAP-t).

Ao isolar a variável IPAg (\%) (expressão 2) e fazer transformações matemáticas, obtém-se a relação simplificada (expressão 7), que permite prognosticar o crescimento valendo-se da seguinte hipótese: "o IPAg potencial de árvores de araucária crescendo livre pode ser prognosticado para os próximos cinco anos em função da variável DAP-t medida no período atual".

$$
\begin{aligned}
& \operatorname{IPAg}(\%)=204,8301 \cdot \text { DAP }_{-t}^{-0,4256} 0,9665^{\text {DAP-t }} \\
& \left\{\left[\left(g_{-} g_{-t}\right) / g_{-t}\right] \cdot 100\right\} / t=204,8301 \cdot \text { DAP }_{-t}^{-0,4256} 0,9665^{\text {DAP-t }} \\
& \left\{\left[\left(g_{-} g_{-t}\right) / g_{-t}\right] \cdot 100\right\}=\left(t \cdot 204,8301 \cdot D P_{-t}^{-0,4256} 0,9665^{\text {DAP-t }}\right) \\
& {\left[\left(g-g_{-t}\right) / g_{-t}\right]=\left[\left(t .204,8301 \cdot \text { DAP }_{-t}^{-0,4256} 0,9665^{\text {DAP-t }}\right) / 100\right]} \\
& \left(g_{-} g_{-t}\right)=g_{-t}\left[\left(t \cdot 204,8301 \cdot \text { DAP }_{-t}^{-0,4256} 0,9665^{\text {DAP-t }}\right) / 100\right] \\
& \mathrm{IPAg}_{\text {potencial }}=\left(\pi \cdot \mathrm{DAP}_{-t}{ }_{-t} / 4\right) \cdot\left[\left(\mathrm{t} \cdot 204,8301 \cdot \mathrm{DAP}_{-\mathrm{t}}^{-0,4256} 0,9665^{\mathrm{DAP}-\mathrm{t}}\right) / 100\right]
\end{aligned}
$$

Em que: IPAg potencial $=$ incremento periódico anual potencial em área transversal, em $\mathrm{cm}^{2}$.ano ${ }^{-1}$; DAP-t $=$ diâmetro à altura do peito no início do período, em $\mathrm{cm} ; \mathrm{t}=$ número de anos considerados.

$\mathrm{Na}$ modelagem do incremento de árvores individuais o ajuste das funções "Potencial-Modificadora" são uma das formas mais comuns usada para descrever o crescimento (DIELER \& PRETZSCH, 2013). Para araucária a parametrização destas funções será testada com a função modificadora já desenvolvida (COSTA et al., 
2015). Estudos para discernir o efeito de fatores individuais sobre o crescimento é essencial (CIENCIALA et al., 2016), principalmente quando estas funções se valem de variáveis independentes de fácil medição a campo, e que se deseja tornar sustentável o uso das florestas (COSTA et al., 2016). Desta forma, a aplicação desses modelos permitiram simular o crescimento das araucárias e determinar alternativas de manejo florestal adequadas segundo a variação nas relações morfométricas e de tamanho e forma das árvores.

\section{CONCLUSÕES}

A equação ajustada descreve o incremento potencial em área transversal de araucária em região de ocorrência natural. A transformação matemática da função ajustada possibilita estimar o incremento futuro de árvores de araucária com crescimento livre, cuja equação pode ser empregada na parametrização de uma função "Potencial-Modificadora".

\section{REFERÊNCIAS}

ALVARES, C. A.; STAPE, J. L.; SENTELHAS, P. C.; GONÇALVES, J. L. M.; SPAROVEK, G. Köppen's climate classification map for Brazil. Meteorologische Zeitschrift, $\quad$ v. 22, p. 711-728, 2013. Disponível em: http://www.dx.doi.org/10.1127/0941-2948/2013/0507 Acesso em: 27 set 2016.

BONTEMPS, J. D.; BOURIAUD, O. Predictive approaches to forest site productivity: recent trends, challenges and future perspectives. Forestry, v. 87, p. $109-128$, 2014. Disponível em: http://www.dx.doi.org/10.1093/forestry/cpt034 Acesso em: 27 set 2016.

BRAGG, D. C. Optimal Diameter Growth Equations for Major Tree Species of the Midsouth. Southern Journal of Applied Forestry, v. 27, p. 5-10, 2003. Disponível em: http://www.srs.fs.usda.gov/pubs/5331 Acesso em: 27 set 2016.

BRAGG, D. C. Potential relative increment (PRI): a new method to empirically derive optimal tree diameter growth. Ecological Modelling, v. 137, p. 77-92, 2001. Disponível em: http://www.dx.doi.org/10.1016/S0304-3800(00)00433-6 Acesso em: 27 set 2016.

CIENCIALA, E.; RUSS, R.; ŠANTRU゚ČKOVÁ, H.; ALTMAN, J.; KOPÁČEK, J.; HŮNOVÁ, I.; ŠTĚPÁNEKF, P.; OULEHLE, F.; TUMAJER, J.; STÅHL, G. Discerning environmental factors affecting current tree growth in Central Europe. Science of The Total Environment, v. 573, p. 541- 554, 2016. Disponível em: http://www.dx.doi.org/10.1016/j.scitotenv.2016.08.115 Acesso em: 27 set 2016.

COOMES, D. A.; ALLEN, R. B. Effects of size, competition and altitude on tree growth. Journal of Ecology, v. 5, p. 1084-1097, 2007. Disponível em: http://www.dx.doi.org/10.1111/j.1365-2745.2007.01280.x Acesso em: 27 set 2016.

COSTA, E. A.; FINGER, C. A. G.; FLEIG, F. D. Influência da posição social nas relações morfométricas de Araucaria angustifolia. Revista Ciência Florestal, v. 26, p. 225 - 234, 2016. Disponível em: http://www.dx.doi.org/10.5902/1980509821116 Acesso em: 27 set 2016. 
COSTA, E. A.; FINGER, C. A. G.; HESS, A. F. Modelo de incremento em área basal para árvores de araucária de uma floresta inequiânea. Pesquisa Florestal Brasileira, v. $35, \quad$ p. 239-245, 2015. Disponível em: http://www.dx.doi.org/10.4336/2015.pfb.35.83.792 Acesso em: 27 set 2016.

DIELER, J.; PRETZSCH, H. Morphological plasticity of European beech (Fagus sylvatica L.) in pure and mixed-species stands. Forest Ecology and Management, v. 295, p. $97 \quad-\quad 108, \quad 2013 . \quad$ Disponível em: http://www.dx.doi.org/10.1016/j.foreco.2012.12.049 Acesso em: 27 set 2016.

HASENAUER, H. Dimensional relationships of open-grown trees in Austria. Forest Ecology and Management, v. 96, p. 197-206, 1997. Disponível em: http://www.dx.doi.org/10.1016/S0378-1127(97)00057-1 Acesso em: 27 set 2016.

HASENAUER, H. Sustainable Forest Management: Growth Models for Europe. Springer-Verlag, Berlin, 2006. 398 p.

HIGUCHI, P.; SILVA, A. C.; ALMEIDA, J. A.; BORTOLUZZI, R. L. C.; MANTOVANI, A.; FERREIRA, T. S.; SOUZA, S. T.; GOMES, J. P.; SILVA, K. M. Florística e estrutura do componente arbóreo e análise ambiental de um fragmento de Floresta Ombrófila Mista Alto-montana no município de Painel, SC. Ciência Florestal, Santa Maria, v. 23, n. 1, p. 153 - 164, 2013. Disponível em: http://www.dx.doi.org/10.5902/198050988449 Acesso em: 27 set 2016.

HIGUCHI, P.; SILVA, A. C.; FERREIRA, T. S.; SOUZA, S. T.; GOMES, J. P.; SILVA, K. M.; SANTOS, K. F. Floristic composition and phytogeography of the tree component of Araucaria Forest fragments in southern Brazil. Brazilian Journal of Botany, São Paulo, v. 35, n. 2, p. 145-157, 2012. Disponível em: http://www.dx.doi.org/10.1590/S0100-84042012000200004 Acesso em: 27 set 2016.

IBGE - INSTITUTO BRASILEIRO DE GEOGRAFIA E ESTATÍSTICA. Manual técnico da vegetação brasileira. 2ed. Manuais Técnicos em Geociências, 1. Rio de Janeiro, RJ, $\quad$ Brasil, 2012. Disponível em: http://biblioteca.ibge.gov.br/visualizacao/livros/liv63011.pdf Acesso em: 27 set 2016.

IRIARTE, J.; BEHLING, H. The expansion of Araucaria forest in the southern Brazilian highlands during the last 4000 years and its implications for the development of the Taquara/Itarare Tradition. Environmental Archaeology, v. 12, n. 2, $\quad$ p. $115 \quad$ - $127,2007 . \quad$ Disponível em: http://www.dx.doi.org/10.1179/174963107x226390 Acesso em: 27 set 2016.

KING, G. M.; GUGERLI, F.; FONTI, P.; FRANK, D. C. Tree growth response along an elevational gradient: climate or genetics? Oecologia, v. 173, p. 1587-1600. 2013. Disponível em: http://www.dx.doi.org/10.1007/s00442-013-2696-6 Acesso em: 27 set 2016.

NEGRINI, M.; HIGUCHI, P.; SILVA, A. C.; SPIAZZI, F. R.; JUNIOR, F. B.; VEFAGO, M. B. Heterogeneidade Florístico-Estrutural do Componente Arbóreo em um Sistema de Fragmentos Florestais no Planalto Sul Catarinense. Revista Árvore, Viçosa, v. 
38, n. 5, p. 779 - 786, 2014. Disponível em: http://www.dx.doi.org/10.1590/S010067622014000500002 Acesso em: 27 set 2016.

OLIVEIRA-FILHO, A. T.; BUDKE, J. C.; JARENKOW, J. A.; EISENLOHR, P. V.; NEVES, D. R. M. Delving into the variations in tree species composition and richness across South American subtropical Atlantic and Pampean forests. Journal of Plant Ecology, p. 1 - 23, 2013. Disponível em: http://www.dx.doi.org/10.1093/jpe/rtt058 Acesso em: 27 set 2016.

OLIVEIRA-FILHO, A. T.; FONTES, M. Patterns of floristic differentiation among Atlantic forests in Southeastern Brazil and the influence of climate. Biotropica, v. 32, n. 4, p. 793 - 810, 2000. Disponível em: http://www.dx.doi.org/10.1646/00063606(2000)032[0793:POFDAA]2.0.CO;2 Acesso em: 27 set 2016.

PAULSEN, J.; WEBER, U. M.; KÖRNER, C. Tree growth near tree line: Abrupt or gradual reduction with altitude? Arctic, Antarctic and Alpine Research, v. 32, p. 14-20, 2000. Disponível em: http://www.dx.doi.org/10.2307/1552405 Acesso em: 27 set 2016.

PRETZSCH, H.; FORRESTER, D.; RÖTZER, T. Representation of species mixing in forest growth models. A review and perspective. Ecological Modelling, v.313, p. 276-292, 2015.2 Disponível em: http://www.dx.doi.org/10.1016/j.ecolmodel.2015.06.044 Acesso em: 27 set 2016.

QUICKE, H. E.; MELDAHL, R. S.; KUSH, J. S. Basal area growth of individual trees: a model derived from a regional longleaf pine growth study. Forest Science, Bethesda, v. 40, n. 3, p. 528-542, 1994. Disponível em: http://auburn.edu/academic/forestry_wildlife/clpe/Pubs/quickeforestscience.pdf Acesso em: 27 set 2016.

R DEVELOPMENT CORE TEAM R: A language and environment for statistical computing. R Foundation for Statistical Computing, Vienna, Austria. 2013. ISBN 3-900051-07-0, Disponível em: http://www.R-project.org Acesso em: 27 set 2016.

SILVA, A. C.; HIGUSHI, P.; AGUIAR, M. D.; NEGRINI, M.; FERT NETO, J.; HESS, A. F. Relações florísticas e fitossociologia de uma Floresta Ombrófila Mista Montana secundária em Lages, Santa Catarina. Ciência Florestal, Santa Maria, v. 22, n. 1, p. 193 - 206, 2012. Disponível em: http://www.dx.doi.org/10.5902/198050985091 Acesso em: 27 set 2016.

SKOVSGAARD, J. P.; VANCLAY, J. K. Forest site productivity: a review of spatial and temporal variability in natural site conditions. Forestry, v. 86, p. 305-315, 2013. Disponível em: http://www.dx.doi.org/10.1093/forestry/cpt010 Acesso em: 27 set 2016.

VANCLAY, J. K. Modelling forest growth and yield: applications to mixed tropical Forests. Copenhagen: CAB International, 1994. 312 p.

WEISKITTEL, A. R.; HANN, D. W.; JR. KERSGAW, J. A.; VANCLEY, J. K. Forest Growth and Yield Modeling. Wiley-Blackwell, Chichester, 2011. 415 p. 